\title{
Translocation of a Copper 7 intra-uterine contraceptive device with subsequent penetration of the caecum: Case report and review
}

\author{
Pranab Sarkar, MRCOG, MOG, MFFP, Locum Consultant in Obstetrics and Gynaecology \\ Correspondence: Pranab Sarkar, Locum Consultant in Obstetrics and Gynaecology, Department of Obstetrics and \\ Gynaecology, Queen's Park Hospital, Blackburn, BB2 3HH, UK.
}

(Accepted March 6 $\left.6^{\text {th }}, 2000\right)$

\begin{abstract}
Summary
A case of translocated Copper 7 intra-uterine contraceptive device with impending caecal penetration is reported and discussed.

Key words

failed laparoscopy, impending caecal penetration, intrauterine device, perforation and migration, retrieval via laparotomy
\end{abstract}

\section{Key message points}

- A migrating device carries a real potential for visceral perforation with serious consequences.

- A plain X-ray of abdomen and pelvis and an ultrasound scan of the pelvis may be needed for exact localisation of a missing IUD.

- Forceful laparoscopic retrieval, as may risk visceral perforation and fistulla formation, should be avoided.

- A laparotomy may be the safer option to remove a device embedded in the bowel wall.

\section{Case report}

A 29-year-old woman, para 3+1, was referred to the antenatal booking clinic by her GP following 13-weeks amenorrhoea. A Copper 7 intra-uterine contraceptive device (IUD) had been inserted 3 years previously following termination of her last pregnancy. The IUD thread was not seen at her booking visit. Her uncomplicated pregnancy proceeded to a spontaneous vertex delivery of a live girl following spontaneous labour at term. The placenta was delivered by controlled cord traction. The IUD was not expelled with the placenta. The uterine cavity was not explored.

The woman was asymptomatic at follow up 4 weeks later. Examination confirmed that the IUD was not located inside the uterine cavity. An abdominal and pelvic X-ray showed the IUD positioned over the sacrum just to the right of the midline and outside the uterus.

Four weeks later she was admitted for laparoscopic removal of the IUD. However the translocated IUD was not found at laparoscopy. A repeat ultrasound scan (transabdominal and vaginal) and an X-ray of the pelvis showed the IUD located on the right side of the pelvis outside the uterus.

In view of previous failed laparoscopy, a laparotomy was performed. The IUD was not easily seen. On palpation, it was found to be lying right lateral to the caecum and was completely embedded in muscular layer after penetrating the serosal surface. The endothelial layer was not breached. The appendix was absent from a previous operation. The device was removed with fine dissection and the caecal wall repaired in layers.
Following an uneventful post-operative recovery, she was able to go home on the fourth post-operative day. At follow up 6 weeks later she was fine. The IUD culture showed E.Coli sensitive to augmentin, cephalexin, ciprofloxacin and gentamycin. Accordingly, she was treated with a course of augmentin.

\section{Discussion}

Uterine perforation remains the most serious complication of the IUD, occurring in approximately one in 1000 cases. $^{1,2}$ Although the exact mechanism of migration of an IUD after uterine perforation remains unknown, there have been a few reports of serious consequences, including bowel perforation requiring bowel resection and anastomosis. ${ }^{3-5}$

Although bowel and other visceral perforation is rare, the present case highlights the fact that a migrating IUD carries a real potential for bowel perforation. The present case also highlights the difficulty that can be encountered with localisation of a missing IUD. A plain X-ray of the abdomen and pelvis and an ultrasound scan of the pelvis (in particular a transvaginal scan) may be needed to help locate the device prior to retrieval.

It is generally agreed that laparoscopic retrieval should be the preferred method when an IUD is translocated inside the abdominal cavity. ${ }^{1,6}$ However, laparoscopic retrieval may prove difficult when a device is firmly adherent to the actual bowel wall or surrounding tissues. ${ }^{1}$ Also, an embedded device may not be visualised through the laparoscope. If it is visualised, then care is needed to remove the device, as forceful retrieval via laparoscope may risk visceral perforation and fistulla formation. In the presence of adhesions, especially with the copper devices, a laparotomy is fully justified and also may be safer. ${ }^{1,5}$

\footnotetext{
Acknowledgement

Sincere thanks to $\mathrm{Mr} \mathrm{D}$ Goodall, Consultant Obstetrician and Gynaecologist, Queen's Park Hospital, Blackburn, for permission to publish this case.

Statements on funding and competing interests

Funding. None.

Competing interests. None.

References

Guillebaud J (1999). Contraception: Your Questions answered. Third edition, pp363. London: Churchill Livingstone.

Browning JJ, Bigrigg MA. Recovery of the intrauterine device from sigmoid colon. Three case reports. Br J Obstet Gynaecol 1988; 95: 530-532.

Chen CP. Hsu TC, Wong W. Ileal perforation by a Multiload-Cu 375 intrauterine contraceptive device. A case report with review of the literature. Contraception 1998; 58(5): 295-304.

Zakin D, Stern WZ, Rosenblatt R. Complete and partial uterine perforation and embedding following insertion of intrauterine contraceptive devices. 1. Classification, complications, mechanism, incidence and missing string. Obstet Gynecol Surv 1981; 36: 335-353. McKenna PJ, Myllote M J. Laproscopic removal of translocated intrauterine contraceptive device. Br J Obstet Gynaecol 1982; 89: 163-165.

Ghosh A, Ganguly G. Laparoscopic removal of perforated copper-T. TheThird International Scientific Meeting of the RCOG, 12-15 March 1996 New Delhi.
} 